\title{
Decoupling the Influence of Wall Thinning and Cladding Thickness Variations in Pulsed Eddy Current using Principal Component Analysis
}

\author{
Faris Nafiah, Mohammad O. Tokhi, Senior Member, IEEE, \\ Gholamhossein Shirkoohi, Member, IEEE, Fang Duan, Member, IEEE, Zhanfang Zhao, and Owen \\ Rees-Lloyd.
}

\begin{abstract}
Corrosion may develop and grow on steel pipes under layers of insulation and cladding. Inspection of the pipes through these protective layers is of paramount importance. Pulsed eddy current (PEC) is a primary non-destructive testing (NDT) technique candidate for this type of inspection as it requires no contact with the inspection material. To overcome the variability in PEC signals due to variations in the cladding thickness, a large measurement set is analysed in this paper using principal component analysis (PCA). The PCA approach decomposes the signal set into a number of uncorrelated variables that explain the maximum amount of the variance in the data set, in which, in this respect, efficiently separate the influences contributed by the difference in the material properties of cladding and pipe wall. The feasibility of using PCA to quantify simulated steel pipe wall independent of confounding cladding thickness variations is investigated. It is found that, with sufficient amount of data, the approach can effectively separate the influences contributed by the wall thickness variations from the cladding thickness variations.
\end{abstract}

Index Terms - non-destructive testing, pipe inspection, principal component analysis, pulsed eddy current.

\section{INTRODUCTION}

$\mathbf{P}$ ULSED eddy current (PEC) is a non-destructive testing (NDT) technique within the electromagnetic sector, and is an extension of the conventional eddy current (EC) testing technique. Instead of applying a single frequency for current excitation like EC, PEC exploits the wide-band frequency characteristic of a rectangular excitation current to allow higher depth of penetration. It is known to offer the advantage of non-contact inspection, particularly for aircraft [1]-[4] and pipeline inspection [5]-[8].

In most pipelines, corrosion under insulation (CUI) is a typical corrosion type that grows underneath layers of nonconductive insulation and steel cladding. The multi-layered structure of a pipe, as visualised in Figure 1, adds to the complexity of inspection. PEC offers the potential to detect such corrosion without disassembling the insulation.

This work was supported by Lloyd's Register Foundation.

F. Nafiah is with the School of Engineering, London South Bank University, UK and NSIRC, TWI Ltd., UK (e-mail: nafiahf@Isbu.ac.uk).

Mohammad O. Tokhi, Gholamhossein Shirkoohi, Duan Fang and Zhanfang Zhao are with the School of Engineering, London South Bank University, UK (e-mail: tokhim@lsbu.ac.uk).

Owen Rees-Lloyd is with TWI Ltd., UK (e-mail: owen.reeslloyd@twi.co.uk).

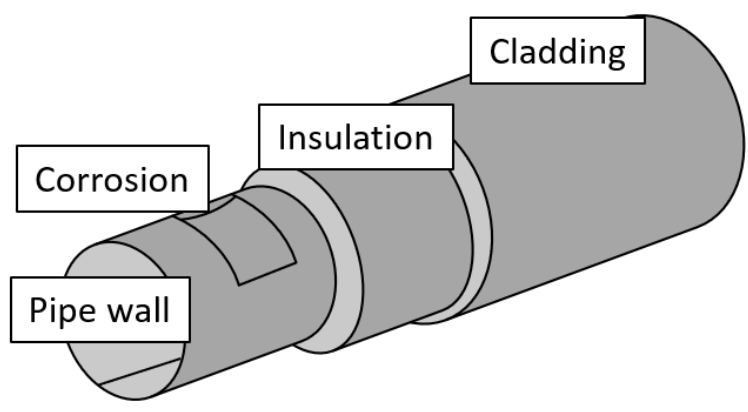

Fig. 1. Representation of corrosion growing under insulation and cladding on a steel pipe.

Recent study in PEC measurement methods, specifically for pipeline inspection, have included the proposition of an inversion method to quantify thickness [8] and estimate the electromagnetic properties of pipe wall [9]. Data collected from a PEC unit has also been used to estimate pipe wall thickness at unmeasured locations by using predictive approach [10]. The utilisation of the time-derivative feature, which offers lift-off immunity, has paved way to the use of regression algorithm to estimate wall thickness [11]. This feature has also been proven useful for wall thickness estimation without insulation 
removal [12], [13]. However, the analysis of cladding thickness variations in the context of pipeline inspection has not been reported.

Principal component analysis (PCA) is a signal decomposition method that transforms large data sets into fewer dimensions that summarise its variance. The employment of PCA is not new in PEC research. Recently, it has been employed for defect characterisation of multi-layered rivet structures [15]. An attempt at comparing PCA with Fast Fourier Transform (FFT) as a method for signal decomposition has also been carried out, which has revealed the benefits of both methods in different aspects for the inspection of heat-treated bearing rings [14]. PCA has also been used for probe parameter analysis for pipeline inspection [16]. Another example that is close to the work carried out here is the application of PCA to separate the signal effects of tubes degradation with those of ferromagnetic support, as reported by Buck et al. [17], owing to the large difference in the "relaxation times" for eddy current diffusion in the tube compared to those of the ferromagnetic support. Similar effort has also been carried out to detect loose parts in steam generator [18] and cracks in inner wing spar of an F/A-18 Hornet aircraft [19].

As briefly mentioned, the discovery of the time-derivative feature affords the immunity towards lift-off and cladding thickness variation; although this is the case, the operators are still expected to manually interpret the raw PEC signals to determine if there is lift-off and cladding thickness variations. In a bid to move towards a more automated process of pipe profiling, this paper explores the reliability of using PCA method to quantify both cladding and pipe wall thickness on a simulated multi-layered pipe structure. Since cladding thickness variation influences the PEC signal differently from the pipe wall variations, PCA signal decomposition is investigated to distinguish between the two distinct signal influences. A similar preliminary study has been done in one of the previous works by using lift-off point of intersection feature to measure coating thickness [20], but the analysis here differs by using PCA. Since this analysis is intended as a feasibility study, the insulation thickness variation is not considered here, as it would add more complexity to the analysis.

The rest of the paper follows the sequential order the problem is approached. Theory, including the working principle of PEC and PCA is introduced in Section II. The experimental setup and development are described in Section III, while Section IV discusses the experimental results. The paper is concluded in SectionV.

\section{THEORY}

\section{A. Pulsed Eddy Current}

PEC extends the capability of conventional EC by supplying a rectangular voltage pulse through the excitation coil, instead of a sinusoidal voltage. The coil, placed above a conductive test sample, induces transient eddy current in the sample. The composite interaction of the eddy current and supply magnetic field is sensed by a separate sensing coil.

Unlike EC, the analysis of PEC response is done by evaluating the transient period of the rising/falling edge of the excitation voltage. Over time, the generated eddy current is diffused to the other side of the sample surface. Simultaneously, due to the sample's resistance, the eddy current also decays. The characteristic diffusion time, $\tau_{D}$, for these eddy currents in a material with $\sigma$ conductivity, $\mu$ permeability, and $d$ thickness is given by [17], [18]

$$
\tau_{D} \sim \mu \sigma d^{2} .
$$

In the case of a multi-layered inspection of CUI on a pipe, the two materials of interest are the carbon steel pipe wall and aluminium cladding. Considering the nominal thicknesses of the materials, the estimated products of $\mu \sigma d^{2}$ are $6.375 \times 10^{8}$ and $7.92 \times 10^{10}$, for aluminium cladding and carbon steel pipe, respectively. The diffusion time for eddy current in the aluminium is of 2 orders of magnitude quicker than the carbon steel. Because of this, besides the longer distance of the probe to the pipe wall's surface as compared to the cladding's, it is expected that the cladding influences the PEC signal at the beginning of the signal, while the pipe wall contributes to the effects at later times.

\section{B. PCA}

PCA is a statistical analysis method of summarising a large set of correlated signal set into a low-dimensional representation of the data (referred to as PCA scores) that describes most of the variability of the original signals. For this work, PCA is applied on a set of PEC signals $\mathbf{V}$, comprising $N$ signals of $P$ discrete samples, obtained by varying the wall and cladding thicknesses. Each signal, $\mathbf{v}_{(n)}$, makes up the row vector of $\mathbf{V}$, where the subscript $n$ represents the respective row in $\mathbf{V}$. Note the bold and uppercase notation of a matrix, and the bold and lowercase notation of a row vector. In most PCA implementations, each row vector is pre-processed by subtracting the row vector from the vector mean (signal average), resulting in the difference signal set $\mathbf{Z}$, composed of row vectors $\mathbf{z}_{(n)}$. The new data set $\mathbf{U}$ after PCA transformation, which is made up of $\mathbf{u}_{(n)}$, has a dimension of $N \times K$, where $K<P$ so that the dimension is reduced. The PCA transformation is carried out by utilising a $P$-dimensional weight vector of the $k$-th component, $\mathbf{w}_{(k)}=\left(w_{1}, \ldots, w_{p}\right)_{(k)}$, using

$$
u_{k(n)}=\left.\mathbf{z}_{(n)} \cdot \mathbf{w}_{(k)}\right|_{\substack{n=1 \ldots N \\ k=1 \ldots K}} .
$$

As previously mentioned, PCA re-organises the data set by order of most variances in the data samples. The value of the first weight $\mathbf{w}_{(1)}$ can be expressed as

$$
\mathbf{w}_{(1)}=\underset{\|\mathbf{w}\|=1}{\arg \max }\left\{\sum_{n}\left(\mathbf{z}_{(n)} \cdot \mathbf{w}\right)^{2}\right\} .
$$

Considering the unit vector property of $\mathbf{w}_{(1)}$, (3) can be rewritten in matrix form as

$$
\mathbf{w}_{(1)}=\arg \max \left\{\frac{\mathbf{w}^{T} \mathbf{Z}^{\top} \mathbf{Z} \mathbf{w}}{\mathbf{w}^{T} \mathbf{w}}\right\} \text {. }
$$

From (4), the term in the curly bracket can be maximised by finding the largest eigenvalue of the matrix $\mathbf{V}(q)^{\top} \mathbf{V}(q)$, which incidentally makes $\mathbf{w}$ to be the corresponding eigenvector. 


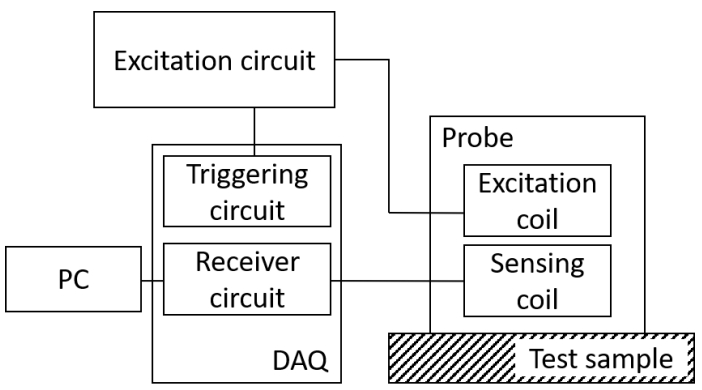

(a)

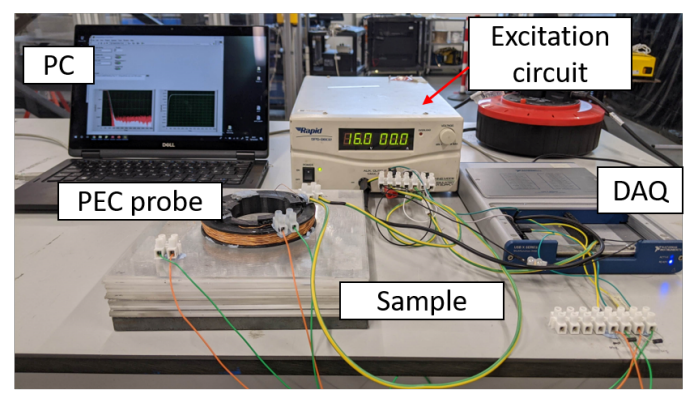

(b)

Fig. 2. (a) Block diagram of the PEC system and (b) experimental setup.

Further $k$-th components can be found by subtracting the first $k$-1 principal components from $W$, resulting in

$$
\hat{\mathbf{Z}}_{(k)}=\mathbf{Z}-\sum_{s=1}^{k-1} \mathbf{Z} \mathbf{w}_{(\mathbf{s})} \mathbf{w}_{(\mathbf{s})}^{\top},
$$

while the weight vector that gives the maximum variance from this new matrix is

$$
\mathbf{w}_{(k)}=\arg \max \left\{\frac{\mathbf{w}^{\top} \hat{\mathbf{Z}}_{(k)}^{\top} \hat{\mathbf{Z}}_{(k)} \mathbf{w}}{\mathbf{w}^{\top} \mathbf{w}}\right\} .
$$

\section{EXPERIMENTAL SETUP}

\section{A. Experiment}

A PEC system as described in Figure 2(a)-(b) was developed for the experimental investigations in this work. The system consisted of three main elements: a probe, an excitation circuit, and a data acquisition (DAQ) system, with a configuration that followed the schematics in Figure 2(a). The probe was a typical transmit-receive circular coil, where an excitation coil was coaxially positioned in the circumference of the sensing coil. The excitation circuit functioned to supply rectangularshaped voltage to the excitation coil, while sending a triggering signal to the DAQ to initiate acquisition. The probe was positioned on top of a thin sheet of aluminium (of $\sigma=25.5$ $\mathrm{MS} / \mathrm{m}$ ), a non-conductive acrylic, and a carbon steel plate (of $\sigma=5.5 \mathrm{MS} / \mathrm{m}$ ) to simulate the multi-layered structure of a pipe. Since this preliminary study was aimed to test the feasibility of the PCA method, the experiment was carried out in a controlled laboratory environment instead.

The probe had the following parameters: excitation coil outer diameter, $d o_{e}=80 \mathrm{~mm}$, excitation coil inner diameter, $d i_{e}=58 \mathrm{~mm}$, excitation coil height, $h_{e}=12.5 \mathrm{~mm}$, sensing coil outer diameter, $d o_{s}=105 \mathrm{~mm}$, sensing coil inner

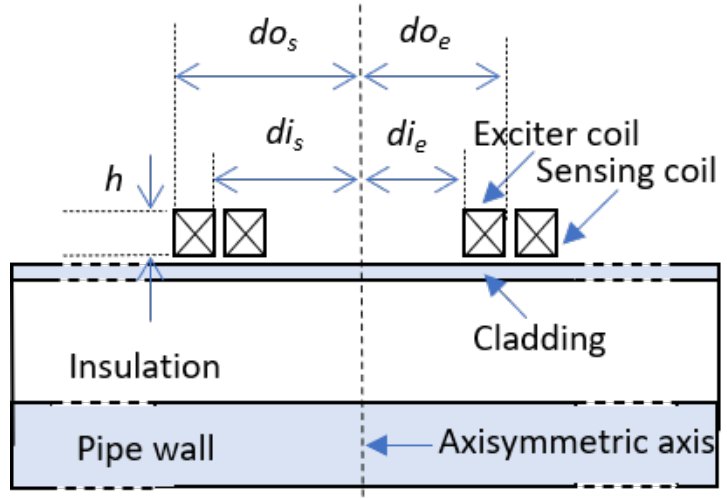

Fig. 3. Cross-sectional image of the experimental setup (not to scale)

diameter, $d i_{s}=83 \mathrm{~mm}$, sensing coil height, $h_{s}=12.5 \mathrm{~mm}$, and sensing coil offset, $O_{s}=2 \mathrm{~mm}$, see Figure 3. The $O$ parameter represents the thickness of the coil former's base. PEC signals were acquired by sensing the potential difference between the two ends of the sensing coil during the rising edge of the $16 \mathrm{~V}, 8 \mathrm{~Hz}$ excitation voltage, with a $50 \%$ duty cycle. During acquisition, 16 signals were acquired and averaged for denoising. The number of turns of each excitation and sensing coil was fixed at 210 .

To simulate wall thinning on the internal surface of the pipe, the carbon steel plate was varied from $4 \mathrm{~mm}$ to $12 \mathrm{~mm}$, at increments of $2 \mathrm{~mm}$. The aluminium sheet was varied from $0.5 \mathrm{~mm}$ to $1.5 \mathrm{~mm}$, at increments of $0.5 \mathrm{~mm}$ to simulate the variations in cladding thickness. The acrylic thickness was fixed at $40 \mathrm{~mm}$. For each thickness change, five sets of signals were obtained for reasons of repeatability.

\section{B. Signal processing}

Data pre-processing is essential prior to PCA, as the diffusion and decay phases of the raw received signals are not derivable in Cartesian domain [16], as seen in Figure 4(a). The raw PEC signals were bi-symmetrically transformed, using

$$
V^{\text {bi-symlog }}(t)=\operatorname{sgn}[V(t)] \cdot \log _{10}\left[1+\mid\left(V(t) \mid / 10^{C}\right],\right.
$$

where $\operatorname{sgn}(*)$ is the standard mathematical Sign (or Signum) function and $C$ is a scaling constant to smoothly transform the region near zero so that it remains finite. In this case, $C$ was chosen as -5 , corresponding to the lowest order observed in the PEC signals. The signal pre-processing amplifies the differences caused by the diffusion of eddy current, represented at only the trailing part of the received signal in Cartesian domain, as can be seen in Figure 4(b).

\section{Results And Discussion}

\section{A. Probe response}

The obtained signals demonstrated the PEC system's sensitivity to the variations of both plate and cladding, as shown in Figure 5. In a glance, the composite interactions between the eddy current decay in the cladding with the plate made it easy to distinguish the effects contributed by either material. To be more specific, the PEC signals of $4 \mathrm{~mm}$ and $6 \mathrm{~mm}$ 


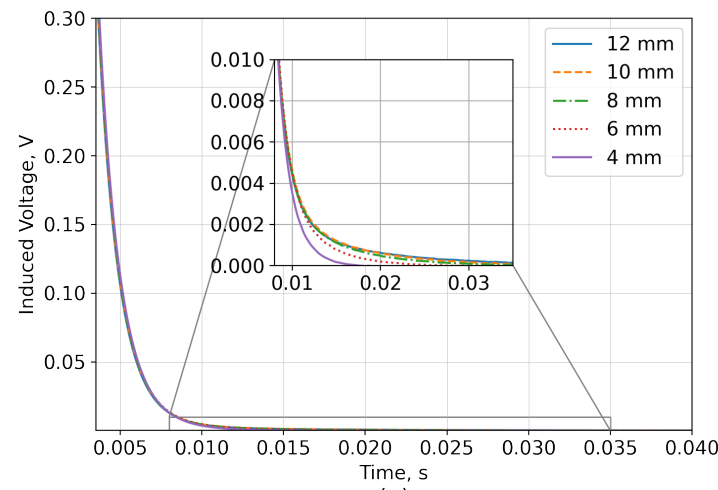

(a)

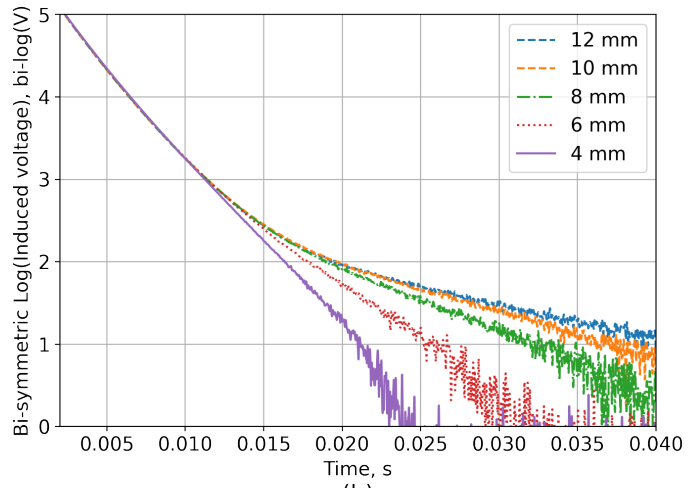

(b)

Fig. 4. Plot of raw PEC signals in a (a) linear plot, and (c) bi-symmetric transformed PEC signals (equivalent to semi-logarithmic plot).

thick samples looked to be more separated in accordance with their corresponding cladding thicknesses, as compared to the $8 \mathrm{~mm}, 10 \mathrm{~mm}$ and $12 \mathrm{~mm}$ signals. For the thick samples, the signal separation corresponding to cladding thicknesses was only more pronounced at the early times $(t<0.02 s)$. This is anticipated, as the product of $\mu \sigma d^{2}$ of the cladding is much lower than its corresponding carbon steel plate, making the influences of the eddy current decay to be noticeable at early times. At the same time, since thin samples have relatively lower $\mu \sigma d^{2}$ values, the PEC signals are highly correlated to the eddy current decay in both the plate and cladding. The eddy current in the cladding diffuses earlier than the plate, resulting in the "temporal shift" effect in the PEC signals only at the early stage.

\section{B. PCA}

A total of 75 measurements were made, making up the test data set containing PEC signals of 6250 data points covering $62.5 \mathrm{~ms}$ acquisition time, for five test repetitions of each of the combinations of plate and cladding thickness variations. Application of PCA to the data set resulted in the first four eigenvectors shown in Figure 6(a) and the subsequent four eigenvectors shown in Figure 6(b).

The first eigenvector accounts for $34.7 \%$ of the variability explained, while the first and second eigenvectors accounts for 92.7\%. When considering the third eigenvector, the cumulative variability explained is $97.0 \%$, while the data set can be explained by its variability up to $98.8 \%$ when considering

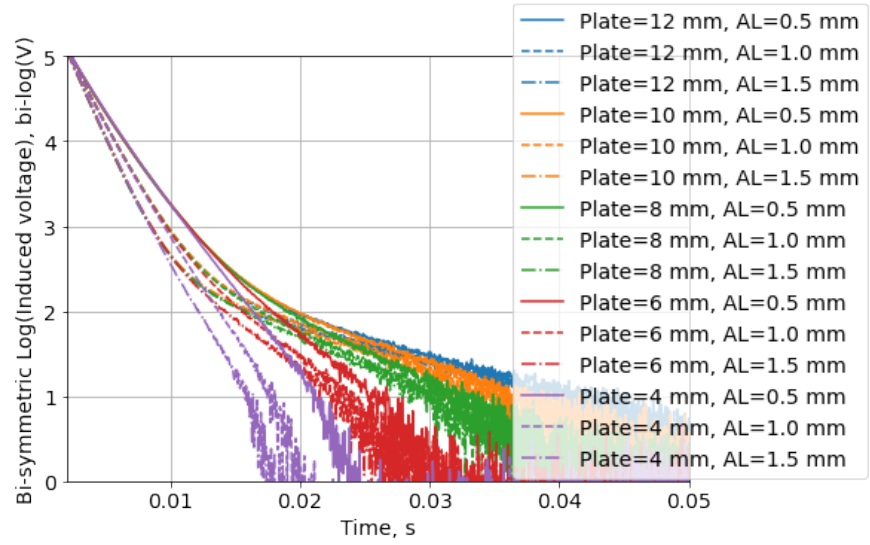

Fig. 5. PEC responses (after bi-symmetric transformation) of different plate and cladding thicknesses.

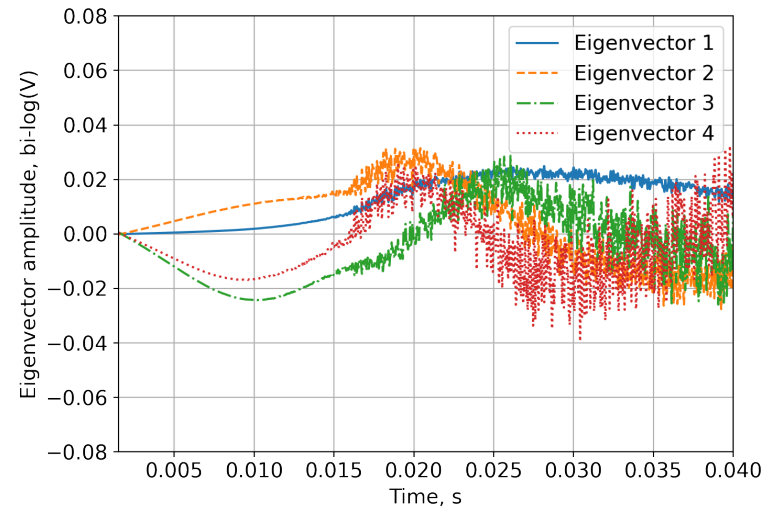

(a)

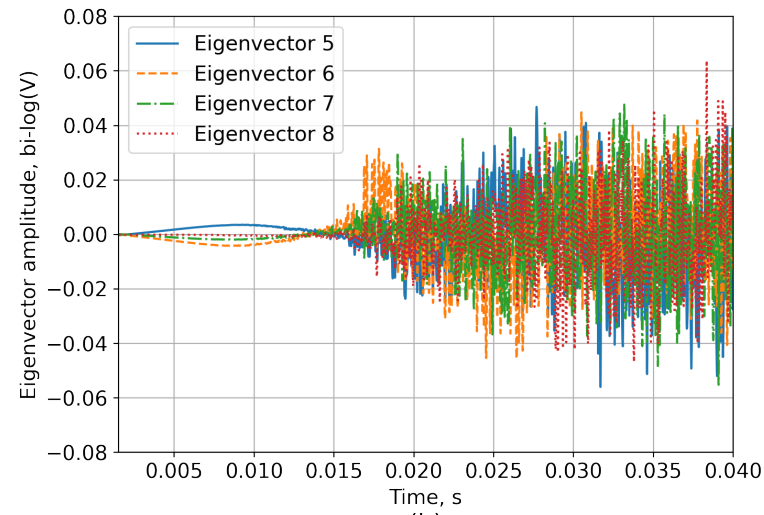

(b)

Fig. 6. Obtained (a) eigenvectors 1-4 and (b) eigenvectors 5-8.

all the first four eigenvectors. Thus, only the first four eigenvectors were considered in the analysis, since the remaining eigenvectors were not significant at the $95 \%$ confidence level. Figure 6(b) also shows that the subsequent eigenvectors after the first four ones were heavily influenced by the noise at the trailing edge of the PEC signals.

By evaluating Figure 6(a), the first eigenvector closely corresponds to the mean of all the bi-symmetric PEC signals, and exhibits a long trailing "tail", which shows its tendency to be chiefly associated with the sample thickness, rather than the cladding thickness. This is explained by the slower diffusion 
time of the carbon steel, which predominantly affects the PEC signals at later times. The second eigenvector, on the other hand, peaks at both $0.02 \mathrm{~s}$ and $0.04 \mathrm{~s}$, with a steady slope at the beginning. Owing to this, the second eigenvector can be claimed to be closely associated with the influences of both cladding and sample thickness. The third eigenvector seems to be the most affected by cladding, where the negative peak value exceeds -0.02 bi- $\log (\mathrm{V})$. However, the same eigenvector also has a peak at time later than $0.02 \mathrm{~s}$, indicating that it is unlikely to contribute to discrimination of cladding and pipe wall thickness. The fourth eigenvector has an almost similar shape with the second eigenvector, except for the "tail" that peaks towards the end of the PEC signals.

The fifth, sixth, seventh and eighth eigenvectors, as shown in Figure 6(b), indicate the inefficiency of the further components in explaining the effects contributed by the sample and cladding thicknesses. Hence, only the first four eigenvectors are considered in subsequent analysis.

From the previous analysis of the eigenvectors, the PCA scores corresponding to each eigenvector can be related to either plate or cladding thicknesses. The first two eigenvectors are plotted against the plate thicknesses in Figure 7(a), while the third and fourth eigenvectors are ploted against the cladding thicknesses in Figure 7(b). Figure 7(a) validates the eigenvector interpretations, demonstrating the relation of the first two eigenvectors with the sample thicknesses. Although the spread of the first scores is higher for $4 \mathrm{~mm}$, its relationship with respect to sample thickness is more linear in comparison to the second scores. This is not particularly surprising, considering the relatively lower peak of the first eigenvector at early times, indicating the higher association of the scores with the plate thickness variations.

Figure 7(b) shows the low association of the third and fourth scores with the cladding thicknesses. Although the corresponding eigenvectors have high peaks at the early times, the trailing data points at the later times are still affected by the plate thickness variations. As a result, the variations in the plate thickness introduce high standard deviation values when attempting to relate the third and fourth eigenvectors with the cladding thickness.

\section{PCA rotation}

As demonstrated in the subsection above, the first two eigenvectors are the most associated with the sample thickness, while the third and fourth eigenvectors can be associated with the cladding thickness. This solution, however, is still limited as the third eigenvector still accounts for the sample thickness, owing to the second peak of the eigenvector at 0.025 s. Motivated by this, the solution can be rotated, so that the variances are redistributed among the rotated components, as was done in previous work [19]. Rotation in PCA is normally done so that each eigenvector can be associated with at most one factor only. In this case, varimax rotation was carried out to maximise the sum of the variances of the squared eigenvectors to represent the cladding thickness solely by the third vector. Since the first four eigenvectors strongly correlate to both sample and cladding thickness variations while the

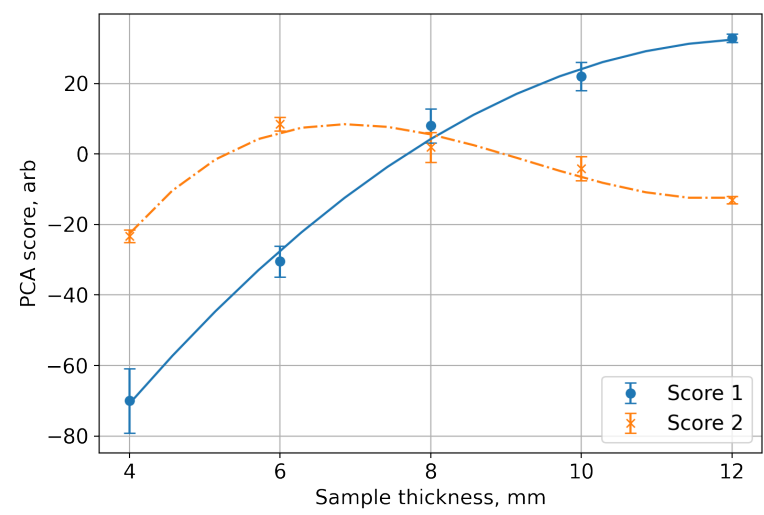

(a)

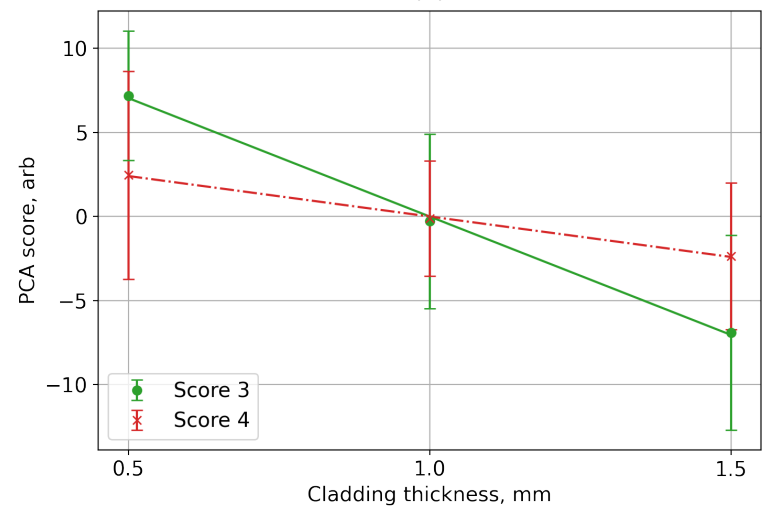

(b)

Fig. 7. (a) First and second PCA scores plotted against plate thicknesses, and (b) third and fourth PCA scores plotted against cladding thicknesses. The errorbar represents the standard deviations.

subsequent eigenvectors are largely influenced by noise, the first four eigenvectors are rotated. This also ensures that the variances are redistributed among the first four scores, without considering the undesirable variances introduced by noise. Apart from limiting the correlation of the cladding thickness with only the third scores, the rotation would conceivably alter the shape of the other eigenvectors.

The rotation matrix of a varimax rotation, $R_{\text {VARIMAX }}$, can be represented as

$$
\begin{aligned}
& R_{V A R I M A X}=\underset{R}{\arg \max }( \\
&\left.\frac{1}{N} \sum_{p=1}^{P} \sum_{n=1}^{N}(v \mathbf{R})_{n k}^{4}-\sum_{p=1}^{P}\left(\frac{1}{N} \sum_{n=1}^{N}(v \mathbf{R})_{n k}^{2}\right)^{2}\right) .
\end{aligned}
$$

where $v$ is the eigenvector unit value, $\mathbf{R}$ is the rotation matrix, and the remaining notations follow the notations for computing PCA as presented in (2)-(4). As previously mentioned, the value of $P$ is less than the dimension of the bi-symmetric PEC signals (in this case, $P=4$ ) so that only the eigenvectors that account for most of the variances of the data are considered.

The new rotated eigenvectors are shown in Figure 8. As a result, the first, second, and fourth eigenvectors are significantly less affected by the cladding thickness variations, judging by the amplitudes of the eigenvectors that reach almost zero before $0.015 \mathrm{~s}$. The first and fourth eigenvectors have the most sample thickness influences, judging by the high peak 


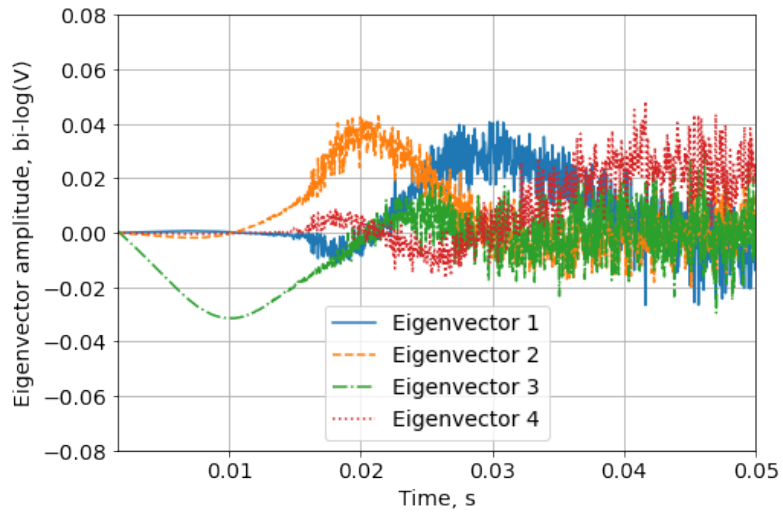

Fig. 8. Rotated first four eigenvectors.

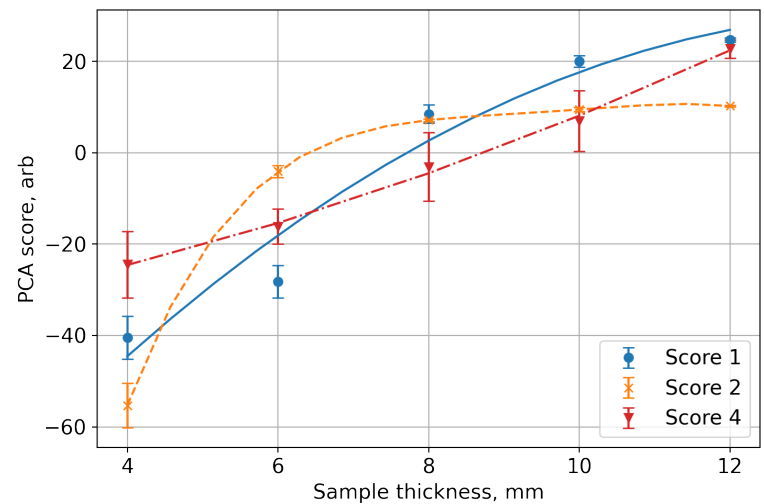

(a)

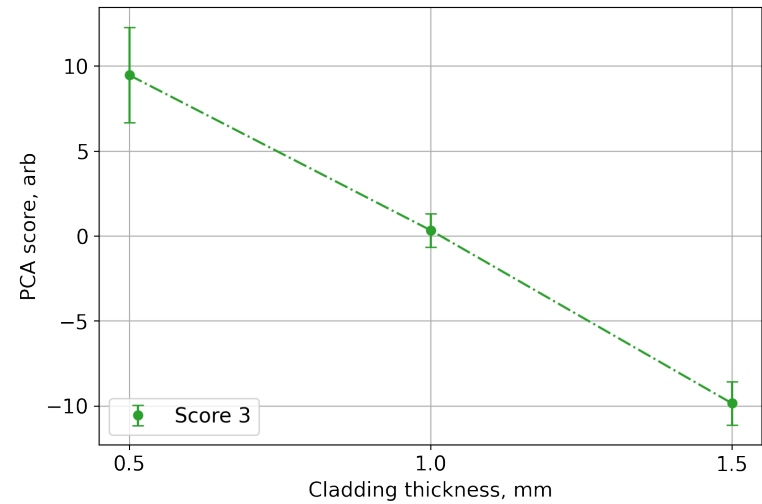

(b)

Fig. 9. (a) First, second and fourth PCA scores plotted against plate thicknesses, and (b) third PCA scores plotted against cladding thicknesses.

values at $0.03 \mathrm{~s}$ and $0.04 \mathrm{~s}$, respectively. It can also be seen that, the effect of the cladding thickness is to introduce an early peak to the third eigenvector, with a marginal height after $0.02 \mathrm{~s}$.

The PCA scores, after eigenvector rotations, are plotted in Figure 9(a)-(b). Different from the previous analysis, the first, second and fourth scores are plotted against the plate thickness in Figure 9(a), following the rotated eigenvectors interpretation. The third PCA scores are plotted against the cladding thickness in Figure 9(b).

The first, second and fourth rotated scores clearly indicte the strong association of the corresponding eigenvectors with the influence of the sample thickness. Another interesting observation is the error bars for both first and second scores for $4 \mathrm{~mm}$ that are relatively longer than the remaining sample thickness. This is because, the PEC signals for $4 \mathrm{~mm}$ decay the fastest, and for this reason, are the most affected by the effects of eddy current diffusion in the cladding. The fourth rotated scores, although still posses linearity with the sample thickness, have a relatively long error bars, indicating the low robustness of the fourth scores to the other influences in the data. In view of the eigenvector direction, it can be noted that the fourth rotated eigenvector is also highly influenced by the noise at the later parts of the PEC signals. From another perspective, the second rotated PCA scores strongly represent the thin plate thickness, but the linearity deteriorates for plates thicker than $8 \mathrm{~mm}$. By analysing the second rotated eigenvector, a peak can be identified at $0.02 \mathrm{~s}$, which explains the strong association of its scores with the thin samples. In contrast, the first and fourth rotated eigenvectors peak at later times, and this effect is strongly demonstrated with its consistent association across the five distinct thicknesses. In the case of quantifying cladding thickness, the third scores were used. It can be seen in Figure 9(b) that the rotated scores efficiently represent the cladding thickness, where there is no visible overlap in the error bars.

\section{Technique limitations}

As noted previously, the conditions of the testing were such that there was substantial data variation. Biases for different cladding and sample thicknesses were purposely introduced, to allow the PCA to favour detecting both cladding and sample thicknesses. The technique could be used as a solution to application-specific problems, such as inspecting pipes with known geometry and properties, where the PCA can be trained prior to the inspection process. With adequate data obtained through various tests, the technique proposed can be used to automate the analysis of PEC signals in discriminating cladding and sample thickness influences. As for an in-situ calibrated PEC process, PCA can only be used in cases where the variations due to both cladding and pipe are severe.

The PCA was further trained for cases with limited variations in the data set. This second test supplied PCA with data sets containing PEC signals corresponding to $0.5 \mathrm{~mm}$ cladding of $4 \mathrm{~mm}, 6 \mathrm{~mm}, 8 \mathrm{~mm}, 10 \mathrm{~mm}$ and $12 \mathrm{~mm}$ samples, and 1.0 mm cladding of only $8 \mathrm{~mm}$ and $12 \mathrm{~mm}$ samples. The choice of combinations of PEC signals was rather arbitrary, but should serve the purpose of limiting the data set size. The rotated eigenvectors from this data set are shown in Figure 10(a). As can be seen, only the two eigenvectors are of potential interest, i.e. the first and the fifth eigenvectors. The first eigenvector peaks at $0.03 \mathrm{~s}$ with a peak value of $-0.03 \mathrm{bi}-\log (\mathrm{V})$, while the fifth eigenvector peaks at $0.01 \mathrm{~s}$ at $0.027 \mathrm{bi}-\log (\mathrm{V})$. The second eigenvector peaks at $0.02 \mathrm{~s}$, but can be associated with both plate and cladding thicknesses. The remaining eigenvectors are not very useful, since they can be claimed to be highly associated with noise. In contrast to the previous case, the fifth eigenvector endures the most variance due to cladding 


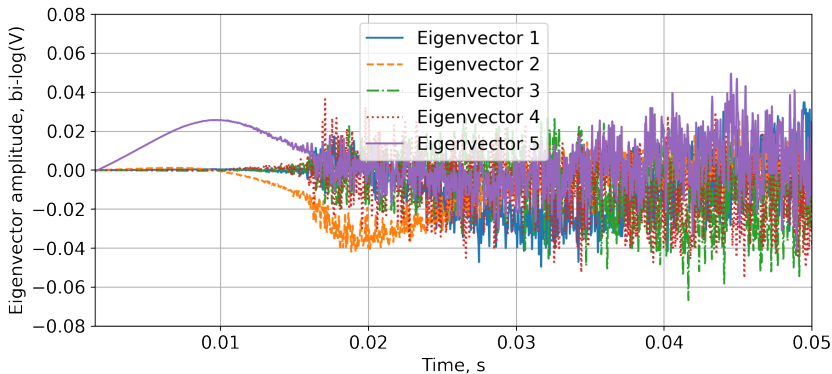

(a)

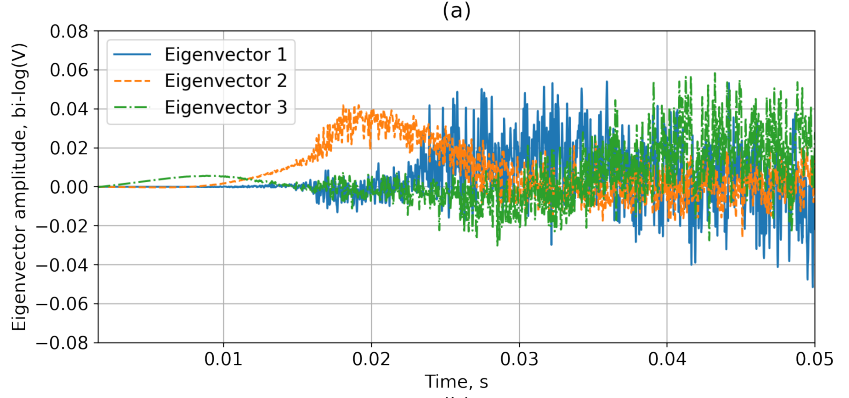

(b)

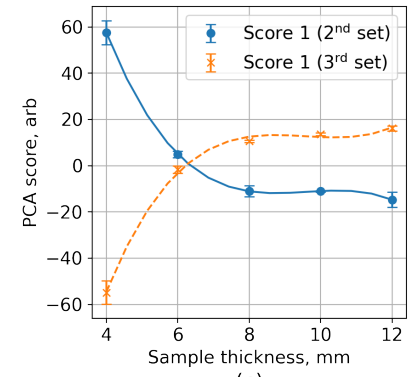

(c)

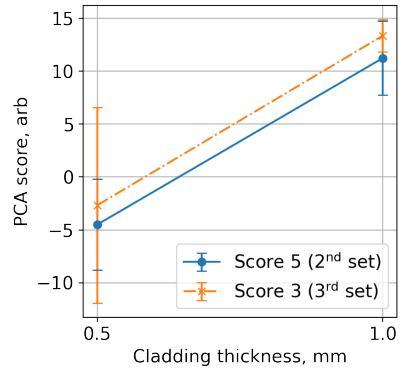

(d)
Fig. 10. (a) First and fifth rotated eigenvector for data set with additional $1.0 \mathrm{~mm}$ cladding for $8 \mathrm{~mm}$ and $12 \mathrm{~mm}$ sample, (b) first and third rotated eigenvector for data set with additional $1.0 \mathrm{~mm}$ cladding for only 12 $\mathrm{mm}$ sample, (c) rotated first scores for both data sets against sample thickness, and (d) rotated first scores for both data sets against cladding thickness.

variations, instead of the third eigenvector. The reason for this is that the new data set is biased towards the sample thickness variations. Nevertheless, the fifth eigenvector still inadequately represents the cladding thickness as the noise in the PEC signals introduced a noisy long "tail" in the eigenvector.

In the case of a more limited variation in the data set, the third test used the data from $0.5 \mathrm{~mm}$ cladding of $4 \mathrm{~mm}, 6 \mathrm{~mm}$, $8 \mathrm{~mm}, 10 \mathrm{~mm}$ and $12 \mathrm{~mm}$ samples, and $1.0 \mathrm{~mm}$ cladding of only $12 \mathrm{~mm}$ sample. The rotated eigenvectors are shown in Figure 10(b). For ease of visualisation, only the first three eigenvectors are shown, since the remaining eigenvectors are only influenced by the high frequency noise at the signal tails. The third rotated eigenvector represents the cladding thickness more poorly, considering the very weak peak at $0.01 \mathrm{~s}$, as compared to another peak at times more than $0.03 \mathrm{~s}$.

The first scores, for both data sets, are shown Figure 10(c) to represent the plate thickness. Notably, both scores show high association with the thickness, and exhibit reliable relationship with the sample thickness. On the other hand, as seen in Figure 10(d), the fifth scores for the second data set separate the two cladding thicknesses, but perform insufficiently. The third scores for the third data set perform much more poorly, where there is visible significant overlaps in the error bars. The third eigenvector, as noted previously, appears to be highly associated with the noise, hence, is unlikely to contribute to discrimination of signals in the presence of different cladding thicknesses.

This further investigation demonstrates the limitation of using the PCA method. Further investigation is evidently necessary, to provide a more comprehensive analysis of this approach, but it can be concluded here that the PCA method undoubtedly needs a large data set to be feasible in separating the signal influences contributed by either plate or cladding. It is also interesting to analyse the practicality of this method on a more accurate representation of an industrial pipe.

The analysis thus far only considers the signal variations introduced by cladding and sample thicknesses. The difference in the diffusion times for the two distinct materials provides significant temporal influences to the PEC signals. However, in the case of insulation thickness variations, the change in the lift-off would introduce amplitude influences to the PEC signals, where thicker insulation contributes to a generally parallel shift in a downward direction, as shown in Figure 11(a).

To test the reliability of using the PCA approach on data
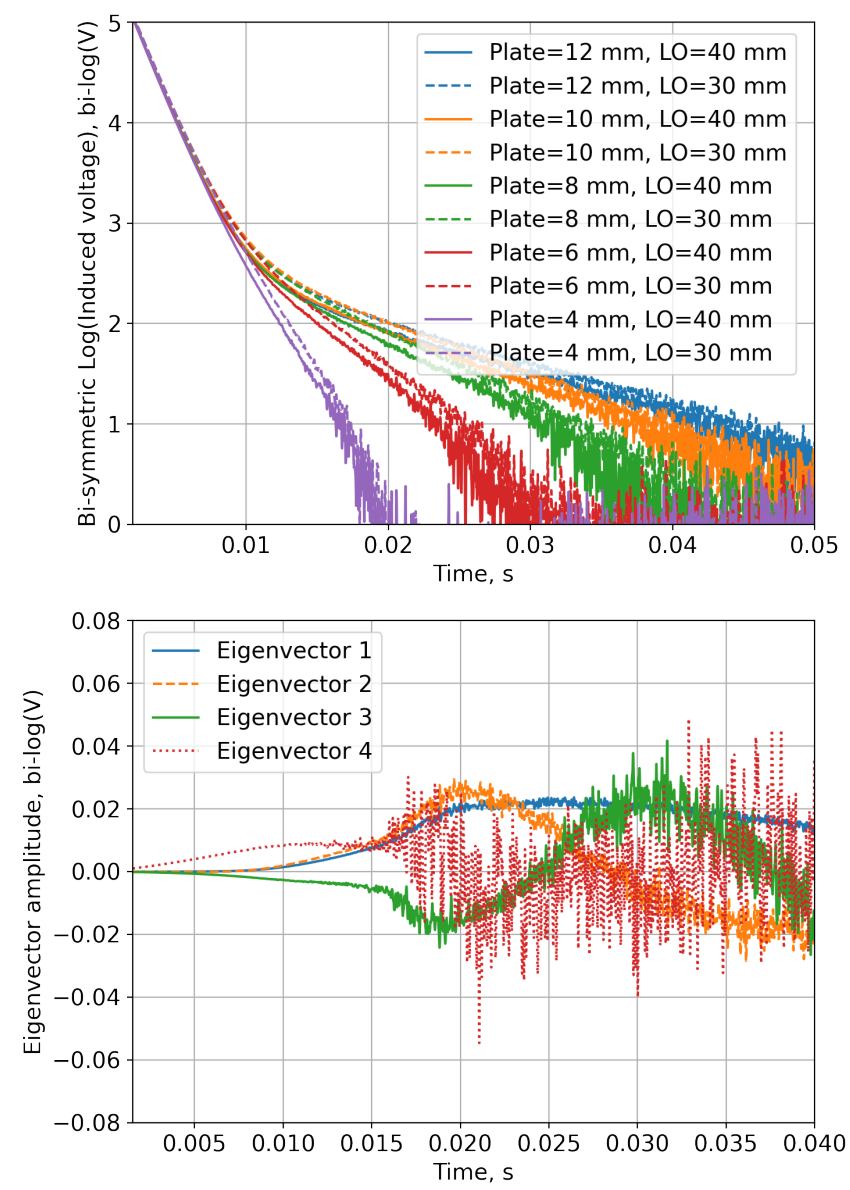

Fig. 11. (a) PEC signals corresponding to different sample and insulation (notated as LO) thicknesses, and (b) their corresponding first four eigenvectors. 


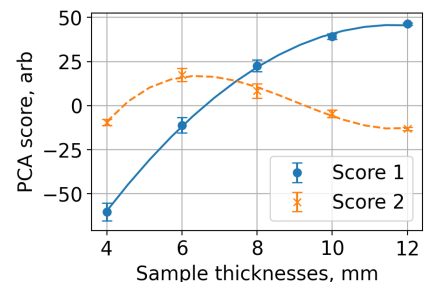

(a)

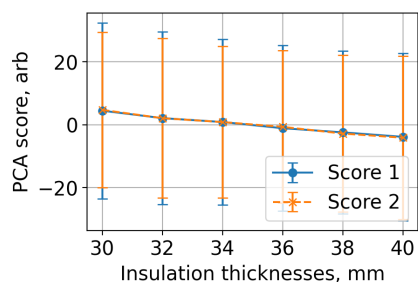

(b)
Fig. 12. (a) Eigenvectors 1-4 plotted against sample thicknesses, and (b) insulation thicknesses.

sets with insulation thickness variations, a second data set was prepared, containing PEC signals corresponding to $4 \mathrm{~mm}, 6$ $\mathrm{mm}, 8 \mathrm{~mm}, 10 \mathrm{~mm}$, and $12 \mathrm{~mm}$ sample thickness, for $30 \mathrm{~mm}$, $32 \mathrm{~mm}, 34 \mathrm{~mm}, 36 \mathrm{~mm}, 38 \mathrm{~mm}$ and $40 \mathrm{~mm}$ insulation thickness. The corresponding eigenvectors after PCA application are shown in Figure 11(b). The shape of the eigenvectors can be claimed to be almost consistent to those in Figure 6(a). The first eigenvector, as with previous solutions, represents the mean of the data set. The second and third eigenvectors are influenced mostly by the late time ranges, while the fourth eigenvector is mostly associated with noise. Unlike previous analysis, the effect of lift-off is the change in amplitude throughout the whole signal length. Since the first eigenvector represents the mean of the data set, this amplitude shift effect is inseparable from the effects contributed by the sample thicknesses. Hence, as seen in Figure 12(a)-(b), the first scores are indicative of sample thicknesses, but not of insulation thicknesses. The second scores are also proportional to the sample thicknesses, but, again, are not strongly associated with the insulation thicknesses, as evidenced by the large value of the standard deviations. The subsequent scores are largely influenced by noises, so they are not shown in the figure.

\section{CONCLUSION}

Multi-layered structure of pipes introduces complexities in interpreting PEC signals, due to the composite interactions of the decay of eddy current in both cladding and pipe wall. This paper has demonstrated that the variations in pipe and cladding have quantifiable responses on PEC signals. Thanks to the difference in the decay rate of different materials of cladding and pipe wall, the PEC signals corresponding to varied plate and cladding thicknesses can be differentiated. PCA has been utilised to further separate the signal influences contributed by pipe wall thickness from cladding thickness. The first, second and fourth PCA scores, after rotation, have been shown to posses the proportional relation with the plate thickness, while the third rotated PCA scores provide the necessary discrimination to separate signals of different cladding thicknesses. However, the method is only applicable if a large data set is available, where biases can be deliberately included.

\section{ACKNOWLEDGMENT}

The authors thank Lloyd's Register Foundation (LRF) for funding this project. LRF helps to protect life and property by supporting engineering related education, public engagement, and the application of research.

\section{RefERENCES}

[1] A. Sophian, G. Y. Tian, D. Taylor, J. Rudlin, "Design of a pulsed eddy current sensor for detection of defects in aircraft lap-joints," Sensors and Actuators, A: Physical, vol. 101, pp. 92-98, 2010.

[2] Y. He, F. Luo, M. Pan, F. Weng, X. Hu, J. Gao, B. Liu, "Pulsed eddy current technique for defect detection in aircraft riveted structures," NDT \& E International, vol. 4, no. 2, pp. 176-181, Mar. 2010.

[3] J. Kim, G. Yang, L. Udpa, S. Udpa, "Classification of pulsed eddy current GMR data on aircraft structures," NDT \& E International, vol. 4, no. 2, pp. 141-144, Mar. 2010.

[4] V.K. Babbar, P.R. Underhill, C. Stott, T.W. Krause, "Finite element modeling of second layer crack detection in aircraft bolt holes with ferrous fasteners present," NDT \& E International, vol. 65, pp. 64-71, Mar. 2014.

[5] W. Cheng, "Pulsed eddy current testing of carbon steel pipes' wallthinning through insulation and cladding," Journal of Nondestructive Evaluation, vol. 31, no. 3, pp. 215-224, Sep. 2012.

[6] Z. Xu, X. Wu, J. Li, Y. Kang, "Assessment of wall thinning in insulated ferromagnetic pipes using the time-to-peak of differential pulsed eddycurrent testing signals," NDT \& E International, vol. 51, pp. 24-29, Sep. 2012.

[7] G. Piao, J. Guo, T. Hu, Y. Deng, H. Leung, "A novel pulsed eddy current method for high-speed pipeline inline inspection," Sensors and Actuators A: Physical, vol. 295, pp. 244-258, Aug. 2019.

[8] C. Chen, J. Li, and Z. Wang, "Inversion Method in Pulsed Eddy Current Testing for Wall Thickness of Ferromagnetic Pipes," IEEE Transactions on Instrumentation and Measurement, vol. 69, no. 12, pp. 9766-9773, Dec. 2020.

[9] C. Chen, and X. Liu, "Pulsed Eddy Current Based Method for Electromagnetic Parameters of Ferromagnetic Materials," IEEE Sensors Journal, vol. 21, no. 21, pp. 6376-6383, Mar. 2021.

[10] L. Nguyen, and J. V. Miro, "Efficient Evaluation of Remaining Wall Thickness in Corroded Water Pipes Using Pulsed Eddy Current Data," IEEE Sensors Journal, vol. 20, no. 23, pp. 6376-6383, Dec. 2020.

[11] N. Ulapane, A. Alempijevic, T. Vidal-Calleja, and J. V. Miro, "Pulsed Eddy Current Sensing for Critical Pipe Condition Assessment," Sensors, vol. 17, no. 10, Sep. 2017.

[12] F. Nafiah, M. O. Tokhi, S. Majidnia, J. Rudlin, Z. Zhao, F. Duan, "Pulsed Eddy Current: Feature Extraction Enabling In-Situ Calibration and Improved Estimation for Ferromagnetic Application," Journal of Nondestructive Evaluation, vol. 39, no. 3, pp. 1-8, Jun. 2020.

[13] N. Ulapane, A. Alempijevic, J. V. Miro, and T. Vidal-Calleja, "Nondestructive evaluation of ferromagnetic material thickness using Pulsed Eddy Current sensor detector coil voltage decay rate," $N D T$ \& E International, vol. 100, pp. 108-114, Dec. 2018.

[14] J. Sha, M. Fan, B. Cao, and B. Liu, "Noncontact and nondestructive evaluation of heat-treated bearing rings using pulsed eddy current testing," Journal of Magnetism and Magnetic Materials, vol. 521, Mar. 2021.

[15] T. Ding, Z. Yang, H. Huang, P. Huang, D. Hou, and G. Zhang, "A method for characterizing defects in multilayer conductive structures by combining pulsed eddy current signals with PCA components," AIP Conference Proceedings, vol. 2102, no. 080003, May. 2019.

[16] F. Nafiah, M. O. Tokhi, G. Shirkoohi, F. Duan, Z. Zhao, G. Asfis, and J. Rudlin, "Parameter Analysis of Pulsed Eddy Current Sensor Using Principal Component Analysis," IEEE Sensors Journal, vol. 21, no. 5, pp. 6897 - 6903, Mar. 2021.

[17] J. A. Buck, P. R. Underhill, S. G. Mokros, J. Morelli, V. K. Babbar, B. Lepine, J. Renaud and T. W. Krause, 'Pulsed eddy current inspection of support structures in steam generators," IEEE Sensors Journal, vol. 15, no. 8 , pp. 4305 - 4312, Aug. 2015.

[18] D. P. Johnston, J. A. Buck, P. R. Underhill, J. E. Morelli, and T. W. Krause, "Pulsed Eddy-Current Detection of Loose Parts in Steam Generators," IEEE Sensors Journal, vol. 18, no. 6, pp. 2506 - 2512 Mar. 2018.

[19] P. Horan, P. R. Underhill, and T. W. Krause, "Pulsed eddy current detection of cracks in F/A-18 inner wing spar at large lift-off using modified principal component analysis," NDT\&E International, vol. 55, pp. 21 - 27, Jan. 2013.

[20] Y. Wang, M. Fan, B. Cao, B. Ye, and D. Wen, "Measurement of coating thickness using lift-off point of intersection features from pulsed eddy current signals," NDT \& E International, vol. 116, pp. 1-12, Jul. 2020. 